\title{
Molecular Mechanism of Tumor Cell Immune Escape Mediated by CD24/Siglec-10
}

\author{
Shan-Shan Yin and Feng-Hou Gao* \\ Department of Oncology, Shanghai Ninth People's Hospital, Shanghai Jiao Tong University School of Medicine, Shanghai, \\ China
}

Tumor immune escape is an important part of tumorigenesis and development. Tumor cells can develop a variety of immunosuppressive mechanisms to combat tumor immunity. Exploring tumor cells that escape immune surveillance through the molecular mechanism of related immunosuppression in-depth is helpful to develop the treatment strategies of targeted tumor immune escape. The latest studies show that CD24 on the surface of tumor cells interacts with Siglec-10 on the surface of immune cells to promote the immune escape of tumor cells. It is necessary to comment on the molecular mechanism of inhibiting the activation of immune cells through the interaction between CD24 on tumor cells and Siglec-10 on immune cells, and a treatment strategy of tumors through targeting CD24 on the surface of tumor cells or Siglec-10 on immune cells.

\section{OPEN ACCESS}

Edited by:

Peter Brossart,

University of Bonn, Germany

Reviewed by:

Matthew S. Macauley,

University of Alberta, Canada Lars Nitschke,

University of Erlangen

Nuremberg, Germany

*Correspondence:

Feng-Hou Gao

fenghougao@163.com

Specialty section:

This article was submitted to Cancer Immunity and Immunotherapy,

a section of the journal

Frontiers in Immunology

Received: 24 March 2020 Accepted: 26 May 2020

Published: 17 July 2020

Citation:

Yin S-S and Gao F-H (2020) Molecular Mechanism of Tumor Cell Immune Escape Mediated by CD24/Siglec-10.

Front. Immunol. 11:1324.

doi: 10.3389/fimmu.2020.01324
Keywords: CD24, Siglec-10, tumor immune escape, immune surveillance, immunotherapy

\section{INTRODUCTION}

Normally, the existence of inhibitory receptors or immune checkpoints avoids the injuries caused by excessive immune response, as tumor cells can up-regulate the corresponding immune checkpoints and their ligands and inhibit the activity of immune cells or induce the apoptosis of immune cells, so as to escape the surveillance of the immune system (1). Tumor immune escape is one of the basic characteristics of tumor occurrence and development (2). The treatment of innate immune checkpoints related to tumor immune escape has achieved remarkable success in recent years. Therefore, the identification of innate immune checkpoints is very important in developing cancer treatment. In this regard, programmed cell death ligand 1 (PD-L1), cytotoxic T lymphocyteassociated protein 4 (CTLA-4), CD47, and some other innate immune checkpoints have been found (3-5). And the latest research has indicated that CD24 may be the dominant immune checkpoint in tumors. Its interaction with sialic-acid-binding Ig-like lectin 10 (Siglec-10) can promote tumor immune escape and is expected to become a new target for tumor therapy (6).

The CD24 gene is located on chromosome 6q21 and it encodes a glycosylated protein with 16 potential O-glycosylation and $\mathrm{N}$-glycosylation sites (7). In general, CD24 is expressed on the surface of developing $\mathrm{T}$ and most $\mathrm{B}$ lymphocytes $(8,9)$. It can determine the ability of proliferation and survival of early T cells (10). Glycosylphosphatidylinositol (GPI) is required to bond with CD24 because the latter does not contain a cytosolic domain. That is why CD24 is also known as a heat stable antigen $(7,11,12)$. The CD24 on immune cells adheres to the lipid raft as a cell adhesion molecule, so that it can participate in the transduction of signals such as tyrosine kinase, $G$ protein, etc. $(13,14)$. CD24 is highly expressed in various tumor cells and is related to the occurrence and development, invasion, and migration of tumor cells (15-25). For example, CD24 is seen as a strong and independent molecular marker for the prognosis of ovarian cancer; it is also related to 
the growth and metastasis of breast cancer and may be related to the occurrence and development of pancreatic cancer $(17,26,27)$. The combination of CD24-expressing tumor cells and P-selectin on platelets can promote the excretion of tumor cells from the bloodstream and thus promote their metastasis (28). Another tumor-related mechanism of CD24 is the connection between CD24 and signal factors in the lipid rafts microdomains, such as Src kinase. The Src kinase activated by CD24 may be involved in other mechanisms that cause tumorigenesis (25). For example, CD24 regulates the invasion of tumor cells by suppressing tissue factor pathway inhibitor-2 (TFPI-2) through a Src-dependent manner (29).

The sialic acid-binding immunoglobulin (Ig)-like lectins (Siglecs) are an immunoglobulin-like type I transmembrane protein with different numbers of Ig-like domains (C2 setting domain) and IgV-like domains that recognize the N-terminal of ligands (30). Siglecs have immune receptor tyrosine inhibitory motifs (ITIM) or ITIM-like motifs in cells, and many of them are related to protein tyrosines that contain an $\mathrm{SH} 2$ domain, like phosphatase 1 (SHP-1), and SHP-2 containing $\mathrm{SH} 2$ domain (31). Siglecs can recognize the sialic acidcontaining structure and combine with the sialic acid attached to the glycoconjugates on the cell surface (31). In this siglecs family, ligand recognition results in an induction of accessibility of the cytosolic ITIM tyrosine and the ITIM-like tyrosine to Src family kinases (32). These kinases phosphorylate ITIM tyrosine in the cytoplasm, thereby recruiting tyrosine phosphatases such as SHP-1 or SHP-2, which can attenuate signal transduction $(33,34)$. Although SHP-1 and SHP-2 both belong to protein tyrosine phosphatases which contain the $\mathrm{SH} 2$ domain, they are usually regarded as negative regulators and positive regulators, respectively $(35,36)$. Siglecs can be divided into two groups according to their structure (30). The first group includes Siglec-1 (Sialoadhesin / CD169), Siglec-2 (CD22), Siglec-4 (Myelin-associated glycoprotein / MAG), and Siglec-15, which are structurally conserved in many species, such as mouse and human (30). The second group contains the CD33-related Siglecs, which are different in mouse and human. CD33-related Siglecs in humans are Siglec-3 (CD33),-5,-6,-7,-8,-9,-10,-11,-12,-14, and-16 while in the mouse Siglec-3(CD33), Siglec-E,-F,-G, and-H belongs to this group (30). Siglec-10 has five extracellular Ig-like domains, a transmembrane region, and a cytoplasmic tail containing two ITIM signaling motifs (37). The IgV structural domain of Siglec-10 contains a key arginine residue, which is related to the recognition of sialic acid (30). Siglec-10 is a kind of inhibitory receptor, which expresses widely in immune cells, such as B cells, monocytes, dendritic cells, a small number of NK cells, and a small subset of activated $\mathrm{T}$ cells which inhibit the function of immune cells (38-40).

Siglec-10 binds firmly to CD24 in a sialylation-dependent manner, and CD24 is the main ligand of Siglec-10 (41, 42). When CD24 on tumor cells combines with Siglec-10 on immune cells, it causes the signal cascade of immune cell inhibition, which is mediated by SHP-1/SHP-2 (31). These phosphatases are associated with ITIM, which is in the cytoplasmic tail of Siglec-10. The ITIM region is phosphorylated, thus blocking Toll-like receptor (TLR)-mediated inflammation and activating a series of intracellular signal pathways to achieve effective immunosuppression and promoting tumor immune escape (Figure 1) $(31,43,44)$. Existing studies show that the damage of danger-associated molecular pattern (DAMP)-associated inflammatory responses, which perform as innate immune pattern recognition receptors, can be reduced by the interaction of CD24-Siglec-10 (42). The interaction between CD24 and Siglecs is considered to be the complex of placental immunosuppressive response, and a great number of placental cells and molecular markers have been evaluated for their role in tumor immune escape (41).

\section{Recognition of CD24 by Siglec-10}

Sialic acid is a family of nine-carbon sugars, which usually forms the terminal sugar structures of the glycoproteins and glycolipids on the cell surface (45). They connect carboxy on C-1, and connect the glycan via $\mathrm{N}$ - and O- on C-2 (46). Sialic acids can be linked to glycans by $\alpha 2-3-, \alpha 2-6-, \alpha 2-8-$, and $\alpha$ 2- 9-linkage (46). Different sialic acid derivatives are formed according to the differences among modifications at C-5, which can be divided into four types: $\mathrm{N}$-acetylneuraminic acid (Neu5Ac), Nglycolylneuraminic acid (Neu5Gc), deaminylneuraminic acid, and neuraminic acid (Neu) (47). The diversity within the sialic acid family itself and the linked- glycan, and the variable linkability of sialic acids, enables cells to synthesize and express a great variety of sialoglycans at the cell membrane $(48,49)$. The differences in the structure of each sialoglycan distinguish them from other sialoglycans. CD24 is a severely sialylated glycoprotein that can interact with Siglec-10 to escape immune recognition (45). It is always heavily decorated with $\mathrm{N}$ - and O-linked glycans $(50,51)$. Sialic acid is connected to glycans through $\alpha$ 2-3- and $\alpha$ 2-6-linkage (Figure 1). In the mouse brain, the glycans of CD24 are mainly complex type N-glycans and highly diverse patterns of O-glycans, including mucintype and carrying $\mathrm{O}$-mannosyl glycans $(52,53)$. It was noticed that structural features of sialic acids are important for Siglec binding (54). The sialic acid backbone can be chemically modified at various positions (54). Its chemical modifications of the sialic acid backbone can dramatically increase the binding affinity to a Siglec $(55,56)$. The carboxylic acid is crucial for Siglec binding and hence is left unmodified, but all other positions, ranging from the aglycone $(\mathrm{C}-2)$ to the rest of the backbone (C-3 to $\mathrm{C}-9$ ), can potentially be modified to improve Siglec binding (54). The sialic acid in mouse brain CD24 is mainly NeuAc, and small amounts of NeuGc can be detected at the non-reducing end of mucin CD24 muchtype O-glycans (Figure 1) (57). The type of linkage and type of underlying sugar also affects the recognition of sialic acids (54). Although all Siglecs can recognize sialoglycans, the binding preferences of these receptors vary considerably (54). Siglec10 recognizes the sialic acid ligands carrying $\alpha$ 2-3- or 2-6linkage (Figure 1). Siglec-10 can be attracted by the unique structure of the entire molecule of CD24 when it is binding to receptors. 


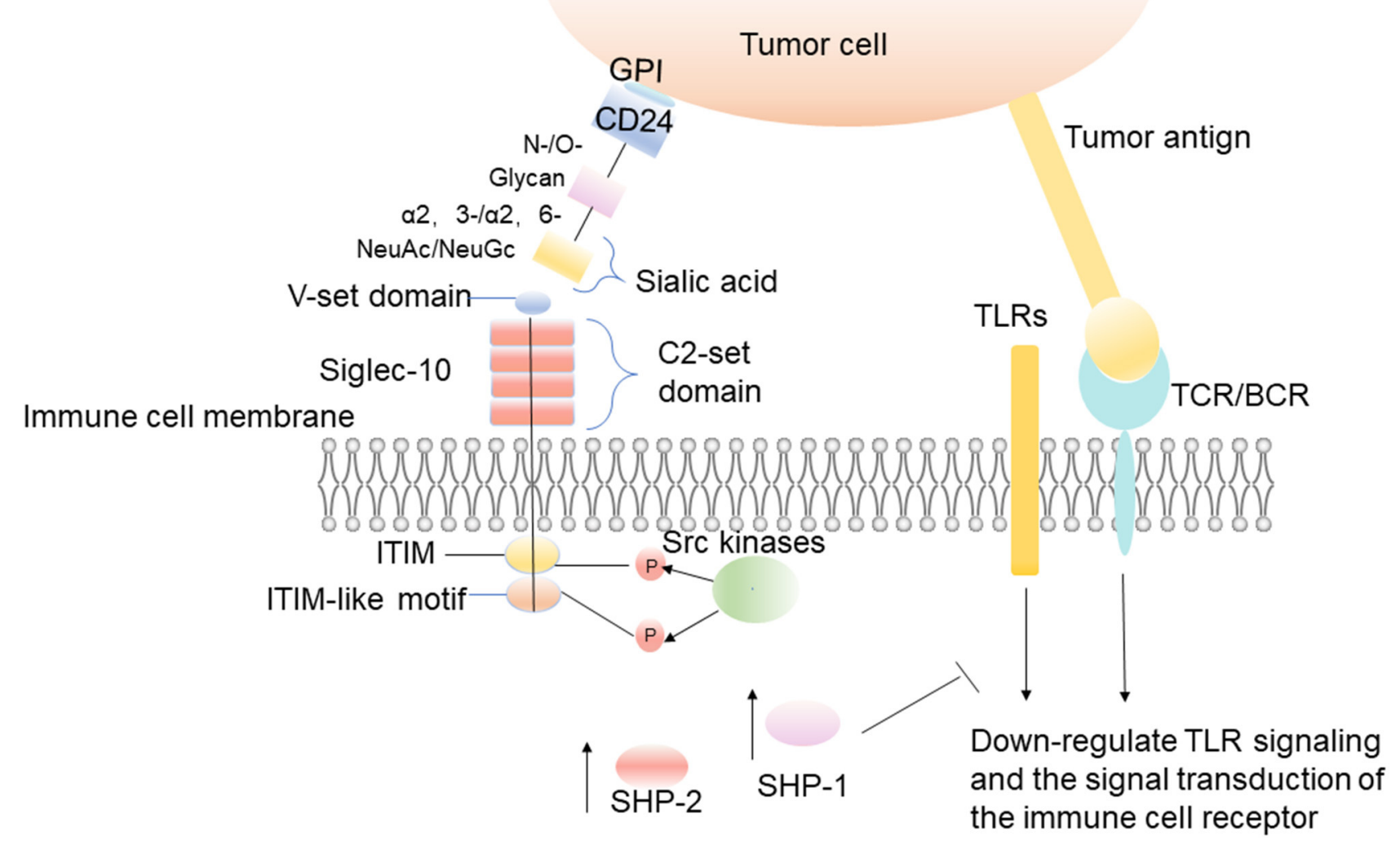

FIGURE 1 | The interaction between CD24 on tumor cells and Siglec-10 on immune cells. The interaction between CD24 on tumor cells and Siglec-10 on immune cells results in inhibitory signal cascades. The lgV domain of Siglec-10 binds to the sialic acid located on the terminal region of CD24, which leads to the induction of Src family kinases by intracellular ITIM or ITIM-like motifs (31). These kinases phosphorylate ITIM tyrosine in the cytoplasm, and then recruit the tyrosine phosphatases such as SHP-1 and SHP-2 to reduce the signal transduction (31).

\section{MOLECULAR MECHANISM OF CD24 EXPRESSION IN TUMOR CELLS \\ CD24 Expression in Tumor Cells Induced by HIF1 $\alpha$}

The tumor cells undergo an exuberant process of metabolism and their oxygen consumption is high. Meanwhile, due to the shortage of oxygen supply, the oxygen content of the tumor microenvironment is low. In this condition, the tumor cells are in a state of relative hypoxia. Hypoxia-inducible factors (HIFs) are the most important proteins for cell-induced expression in hypoxic environments (58). Tumor cells use it to induce the expression of target genes to make tumor cells adapt to the hypoxic environment (59). The main reason for this is that under the action of normal oxygen, the proline hydroxylase hydroxylates those proline residues in the conserved region of HIF subunits, and VHL E3 ubiquitination ligase identifies and ubiquitinates the hydroxylated HIF so that the ubiquitinated HIFs can be degraded rapidly by the proteasome; however, under the condition of hypoxia, the prolinyl hydroxylase of HIF protein is inhibited, which stabilizes the protein level of HIF $\alpha(59,60)$.
Hypoxia-induced CD24 expression mainly occurs at the transcriptional level, especially when HIF acts as a transcription factor to induce CD24 expression (Figure 2). CD24 is the key transcriptional target of HIF-1 $\alpha$ (61). HIF- $1 \alpha$ promotes the transcription of CD24 through a functional hypoxia-responsive element in the CD24 promoter (61). In a previous study based on broad transcriptomic analysis of human umbilical cord vein endothelial cells exposed in vitro to hypoxia, Scheurer et al. reported that CD24 is one of the 65 genes that mRNA increases with hypoxia (65). In bladder cancer, prostate cancer, and gastric cancer, hypoxia significantly up-regulated the expression of CD24 mRNA and protein in cancer cells $(61,66)$.

\section{Non-coding RNA Induces CD24 Expression in Tumor Cells}

Signals from non-coding RNAs (ncRNAs) can transfer between tumor cells and tumor microenvironments through extracellular vesicle (EVs), exosomes, and gap junctions $(67,68)$. NcRNAs have been demonstrated to play an important role in tumor growth, metabolism, and migration, as well as in regulating the expression of CD24. It has been found for the first time that the increased expression of long non-coding RNA (lncRNA) 


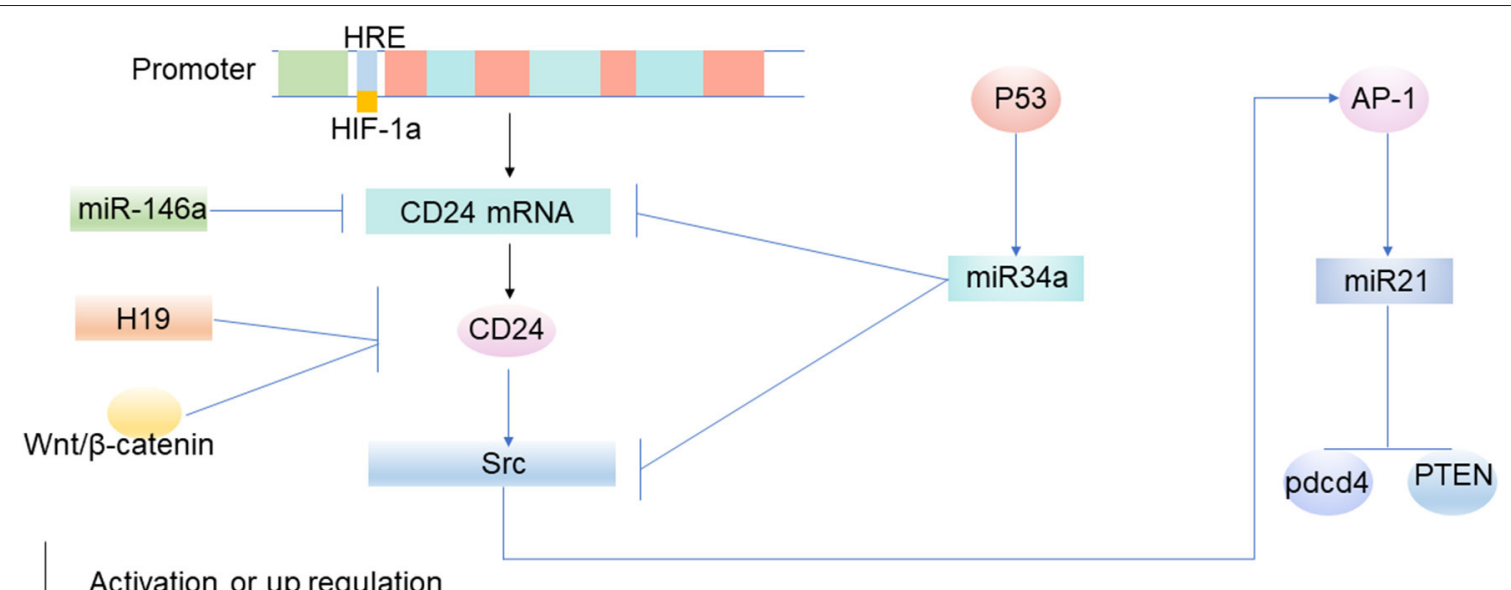

Activation or up regulation

$\perp$ Inhibition or down regulation

FIGURE 2 | The molecular mechanism of CD24 expression in tumor cells. Under the condition of hypoxia, HIF-1 $\alpha$ promotes the transcription of CD24 through a functional hypoxia-responsive element on the promoter of CD24 (61). MiRNA participates in tumorigenesis by participating in the upstream and downstream regulatory networks of CD24, and mainly inhibits the translation of mRNA. MiR-34a targets CD24 and Src at the post-transcriptional level, and inhibits the expression of CD24 and Src (62). When miR-34a is epigenetically silenced, the expression of CD24 up-regulates (62). The upregulation of CD24 expression can increase the expression of miR21 by activating Src, thus inhibiting the expression of Pdcd4 and PTEN, and participating in the occurrence and development of tumors (62-64).

H19 leads to a reduction of cell-surface CD24, and that downregulation of $\mathrm{H} 19$ helps to maintain the expression of $\mathrm{CD} 24$ on the cell surface, so H19 is thought to make a contribution to cell invasion by regulating CD24 expression, thereby regulating tumor immune escape (Figure 2) (69). MiRNA is also a kind of ncRNA. The protein-coding genes, such as the CD24 gene, are currently known to be regulated by miRNAs (62). Mature miRNAs regulate genes in two ways: one way is to bind to the target gene mRNA and promote its degradation, and the other is to inhibit the translation of mRNA (70). MiRNA participates in tumorigenesis by participating in the upstream and downstream regulatory networks of CD24, and mainly inhibits the translation of mRNA. MiR-34a targets CD24 and Src at the post-transcriptional level, and inhibits the expression of CD24 and Src (Figure 2) (62). When miR-34a is epigenetically silenced, the expression of CD24 up-regulates (62). CD24 is the direct target of miR-146a (71). MiR-146a binds to the 3'untranslated region (UTR) of CD24 and suppresses its expression after transcription (Figure 2) (71).

\section{WNT/ $\beta$-catenin Induces CD24 Expression in Tumor Cells}

The Wnt/ $\beta$-catenin signaling pathway is an evolutionarilyconserved regulatory pathway that governs numerous normal cellular and developmental processes such as cell fate determination, cell proliferation, and migration (72). However, aberrant Wnt signaling has also been identified as a key mechanism in cancer biology. It has been proven that Wnt/ $\beta$-catenin plays an important role in tumor growth and regulating the expression of CD24. Immunoprecipitation studies show that $\mathrm{CD} 24$ may activate $\beta$-catenin to interact with the Wnt pathway and induce $\beta$-catenin translocation into the nucleus (72). It has been shown in breast cancer that $\beta$-catenin can inhibit tumor immune escape by down-regulating the expression of CD24 (Figure 2) (73). And it has also been identified that CD24 is the transcriptional target of Wnt signaling in a non-transformed human mammary epithelial cell line MCF 10A (73).

\section{THE INTERACTION BETWEEN SIGLEC-10 ON IMMUNE CELLS AND CD24 INDUCES IMMUNE ESCAPE OF TUMOR CELLS}

\section{T Cells}

Malignant cell-secreted Evs in the tumor microenvironment stimulate lymphocytes to suppress anti-tumor immunity and promote tumor progression. Importantly, malignant EVs impair $\mathrm{T}$ cells' function by upregulating the expression of Siglec-10 on T cells (74). Siglec-10 is an inhibitory receptor expressed on the surface of $\mathrm{T}$ cells $(38,39)$. It triggers immunosuppression by blocking the activation of TCR, which is realized by inhibiting the formation of $\mathrm{T}$ cell major histocompatibility complex class I (MHC-I) peptide complex and the phosphorylation of $\mathrm{T}$ cell receptor-associated kinases Lck and ZAP-70 (Figure 3) (40, 75,76 ). Studies by Bandala-Sanchez et al. have also shown that Siglec-10 expressed on the $\mathrm{T}$ cells' surface inhibits the phosphorylation of $\mathrm{T}$ cell receptor-associated kinase ZAP-70 and the activation of $\mathrm{T}$ cells $(39,75)$. Siglec-10 can also inhibit T cells' activation by binding to corresponding ligands. For example, related studies by Sammar et al. have shown that CD52 (and 


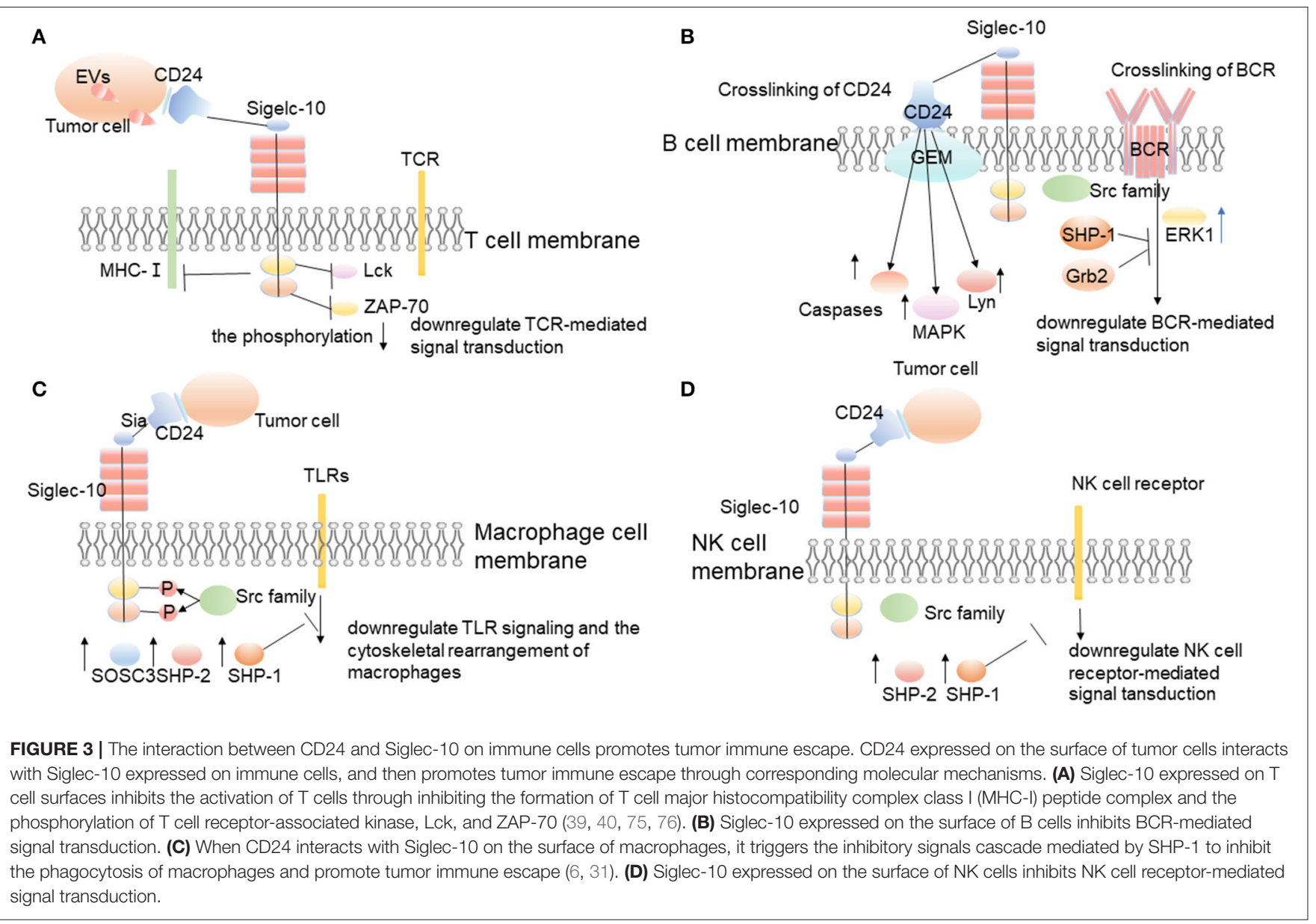

possibly CD24, if properly glycosylated) can bind to Siglec-10 and block the activation of TCR by inhibiting T cell receptor (TCR)related kinases (Figure 3) (41). CD24/Siglec-10 can inhibit the activation of $\mathrm{T}$ cells mediated by TCR and promote tumor immune escape.

\section{B Cells}

Siglecs play an important immunomodulatory role in B cell activation and immunoglobulin production (77). For example, Siglec-2 strongly affects the signal transduction of the B cell receptor $(\mathrm{BCR})$ and has become the prototype of the working principle of Siglec signal transduction $(30,78)$. B1 cells, the subtype of B cells, express the inhibitory receptor Siglec-10 in humans. However, it is still unclear how Siglec-10 regulates the activity of B cells (38). Siglec-10 not only expresses in human B cells, but also in mouse B cells, in which case it is referred to as Siglec-G. Siglec-G is an ortholog of human Siglec-10 (79). They have a high sequence identity, similar chromosomal location of their genes, and conserved structure of the proteins (79). Lineal homologs often have similar or even the same functions, which are regulated by a similar pathway, and play similar or even the same roles in different species (80-82). For example, both Siglec-10 in human cells and Siglec-G in mouse cells can combine with CD24 to inhibit host inflammation and immune response triggered by damage-related molecular models, and Siglec-10/G plays an important role in self-nonself discrimination of the immune system and may be involved in evasion of host immunity by RNA viruses $(42,45)$.

Existing studies have found that Siglec-G is an inhibitory receptor of B cells, which controls the proliferation and calcium signal transduction of B1 cells (83). Siglec-G is expressed in a B cell-restricted way, with large amounts present in B1 cells (83). When overexpressed, Siglec-G can inhibit B cell receptormediated calcium signaling (83). Siglec-G dampens the calcium signal transduction of B1 cells by recruiting the ITIM-binding protein SHP-1, growth factor receptor binding protein 2 (Grb2), and inhibits the activity of transcription factors NFATc1 and NF-kB (Figure 3) (45).

CD24 can also affect the function of B cells by affecting the signal transduction of BCR (Figure 3). CD24 induces human $\mathrm{B}$ cell apoptosis through glycolipid-enriched membrane (GEM) domains / raft-mediated signal transduction systems (12). The recruitment of a variety of signal transduction molecules in the GEM domain, including Src family PTKs, trimer G protein, Ras, and linker for activation of $\mathrm{T}$ cells, indicates their role as a signal transduction platform $(84,85)$. The association of CD24 and lyn protein tyrosine kinase in GEM enhances, and the activity of lyn also enhances, after CD24 cross-linking (12). 
In addition, after CD24 cross-linking, mitogen-activated protein kinases (MAPK) is activated, and CD24 mediates intracellular signal transduction that leads to $\mathrm{B}$ cell apoptosis (Figure 3) (12). The stromal-cell-derived factor-1 (SDF-1, also known as CXCL12) has a strong chemotactic effect on lymphocytes, and chemokine receptor CXCR4 is a specific receptor of CXCL12 (86). Using CD19-positive bone marrow B cells and CD24/-Pre-B lymphocyte lines isolated from CD24 knockout mice proves that the expression of CD24 decreases CXCL12-mediated cell migration and signal transduction through CXCR4 (86). The study results suggest that CD24 mediates the apoptosis of human precursor $B$ cells with the activation of multiple caspases in the pro-B and pre-B stages (87). The cross-linking of BCR precursors causes rapid and strong activation of extracellular signal-regulated kinase 1 (ERK1), while the cross-linking of CD24 induces continuous activation of p38MAPK after the activation of ERK1 (Figure 3) (87). Therefore, it can start the inhibitory signal, play its regulatory role, and promote tumor immune escape.

\section{The Interaction Between CD24 on the Surface of Tumor Cells and Siglec-10 on Macrophages Induces Tumor Immune Escape}

The phagocytosis of macrophages to tumors is regulated by a host of signals, including pro-phagocytosis signals ("Eat me") and anti-phagocytosis signals ("Don't eat me") (88). Many phagocytic signals are expressed on the tumor surface, including tumor-associated antigen, endoplasmic reticulum chaperone, calreticulin, and glycoprotein signal lymphocyte activation molecule family member 7 (SLAMF-7; also known as CD319) (89-91). However, some anti-phagocytic signals also exist on the surface of tumor cells, including CD47, PD-L1, $\beta 2$ microglobulin $(\mathrm{B} 2 \mathrm{M})$, an unidentified ligand that binds to leukocyte immunoglobulin-like receptor-2 (LILRB2), and the recently discovered CD24 (6, 92-95). These "don't eat me" signals interact with the corresponding receptors on phagocytes surface, including signal regulatory protein $\alpha$ (SIRP $\alpha)$, programmed cell death 1 (PD-1), leukocyte immunoglobulin-like receptor 1 (LILRB1), Siglec-10, etc. The interaction between these receptors and ligands promotes the tumor to escape the phagocytosis of phagocytes. These anti-phagocytosis signals are all involved in macrophage signaling based on immunoreceptor-tyrosinebased inhibition motifs and essentially avoid the surveillance and clearance of macrophages (6). The researchers used gene knockout against CD24, Siglec-10, and monoclonal antibodies to block CD24 and Siglec-10, and then they found that macrophages increased their ability to engulf tumors and slow down the growth of macrophage-dependent tumors in vivo (6). And all the macrophages expressing Siglec-10 responded to the blocking of CD24, and the degree of these responses were related to Siglec10. The loss of siglec-10 would decrease the blocking of CD24. It indicates that the specific blocking of CD24 occurs between CD24 and Siglec-10 (6). CD24 binds specifically to Siglec-10 but not to Siglec-3 and Siglec-5 (41). The interaction between CD24 and Siglec-10 triggers the inhibitory signal cascade (Figure 3 )
(45). After SRC family tyrosine kinases phosphorylate the cytoplasmic tyrosine-based signal transduction group, Siglec-10 recruits and activates the proteins containing the $\mathrm{SH} 2$ domain, especially SHP-1, SHP-2, or suppressor of cytokine signaling 3(SOCS3) (Figure 3) (31). As an important member of the tyrosine phosphatase family, SHP-1 can specifically bind to tyrosine phosphorylated in the intracellular ITIM domain and catalyze its dephosphorylation. It can also negatively regulate the intracellular signal transduction in which the growth factors, cytokines, hormones, extracellular matrix, and cell adhesion molecules are involved (96). Therefore, the interaction between CD24 and Siglec-10 inhibits the phagocytosis of macrophages, so that the tumor cannot be cleared by phagocytosis, which promotes the immune escape of tumors.

Healthy normal tissues and cells have the inherent ability to avoid the self-elimination of macrophages by expressing anti-phagocytosis molecules, but cancer cells rely even more on similar mechanisms to escape the eradication of immunemediated (97-100). Therefore, the targeted therapy toward the macrophage phagocytosis checkpoints in tumors may provide a new avenue for the development of cancer immunotherapies to eliminate tumor immune escape (101).

\section{NK Cells}

In liver tumor microenvironments, Siglec-10 mainly expresses on NK cells, while it expresses less on T cells and B cells (102). The percentage of Siglec-10+NK cells in tumor tissues is higher than that in surrounding non-tumor tissues (40). The high expression of Siglec-10 on NK cells can mediate the functional damage of NK cells in human hepatocellular carcinoma (HCC) (40). According to the results of survival analysis, the increased expression of Siglec-10 in HCC is negatively correlated with the prognosis of patients with HCC (40). The interaction between CD24 and Siglec-10 can repress the tissue damage-induced immune responses (42). And the interaction between CD24 expressed by hepatoma cells and Siglec-10 expressed by NK cells may be beneficial for tumors to escape the killing effect of NK cells and promote tumor immune escape (Figure 3) (40).

\section{THE STRATEGY OF TARGETING CD24/SIGLEC-10 TO INHIBIT TUMOR IMMUNE ESCAPE}

\section{Antibodies of Targeting CD24}

SWA11 monoclonal antibody has high affinity and specificity toward CD24-expressing cells (21). CD24 is internalized in cells after the binding of SWA11mAb, and the role of the SWA11 monoclonal antibody is mainly to reduce the proliferation of tumor cells $(21,103)$. SWA11mAb targeting CD24 effectively retarded the growth of lung and ovarian carcinoma xenografts (103). Dual treatment of pancreatic adenocarcinoma cells with anti-CD24 mAb and cetuximab enhanced phagocytosis relative to either treatment alone, demonstrating a potential synergy between anti-CD24 mAb and anti-solid-tumor mAbs. Besides, the addition of anti-CD24 antibody to the chemotherapy regimen may be beneficial to target chemotherapy-resistant tumor stem 


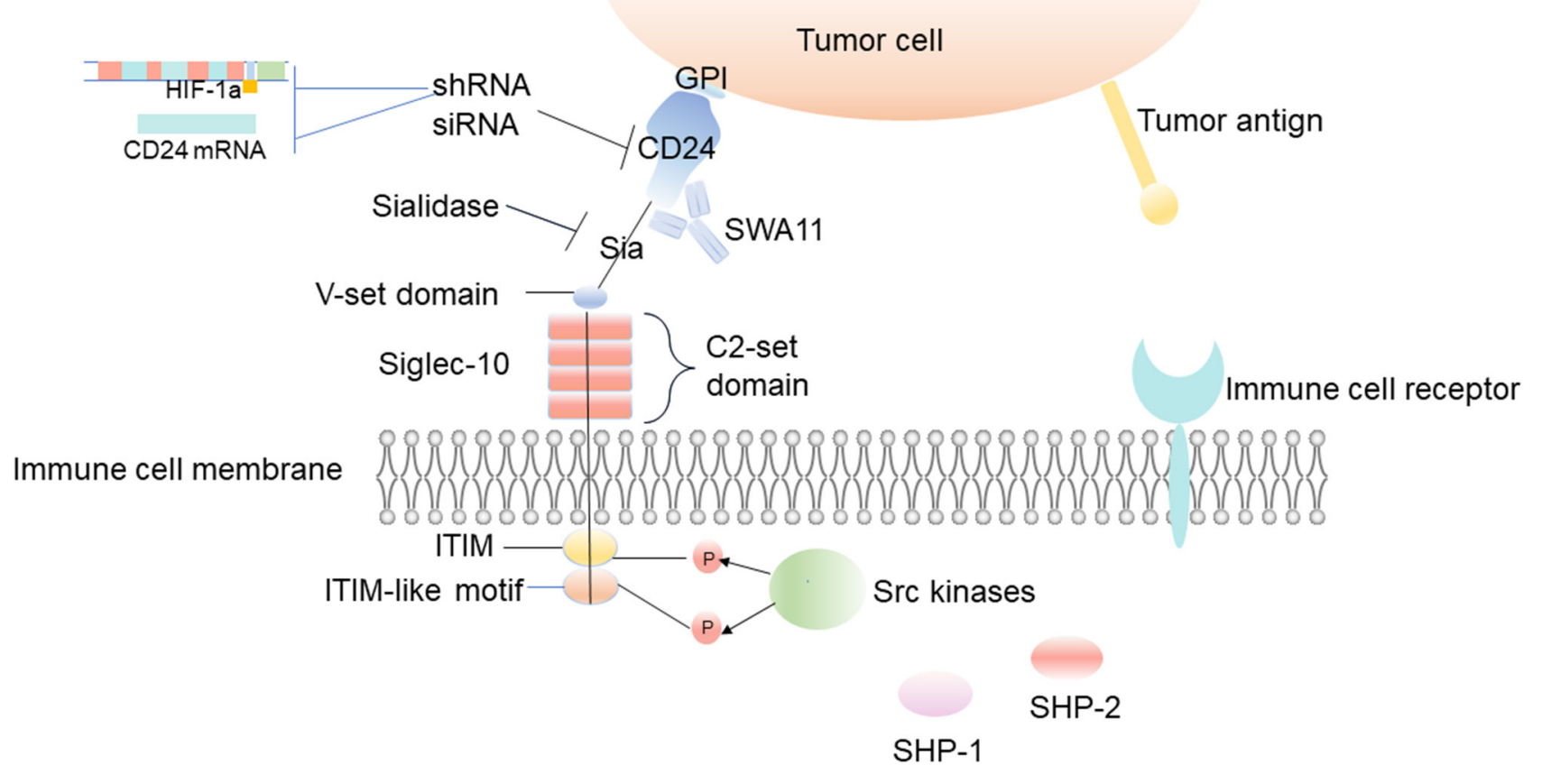

FIGURE 4 | Inhibition of tumor immune escape by interfering with CD24/Siglec-10 signal transduction. The uses of CD24 monoclonal antibodies or sialidase is designed to interfere with the interaction between CD24 and Siglec-10, block the transduction of inhibitory signals, and inhibit the immune escape of the tumor. And the decrease of $\mathrm{HIF}-1 \alpha$ or CD24 expression is also a potential method for the treatment of inhibiting the immune escape of tumors.

cells (103). However, potential off-target effects of anti-CD24 $\mathrm{mAb}$ treatment in humans include the depletion of B cells, owing to high CD24 expression by B cells (6). Meanwhile, the potential toxicity of targeted CD24 to cancer patients cannot be ruled out at this stage (6).

\section{Targeting CD24 and Siglec-10 Genes}

Through gene ablation of CD24 and Siglec-10, the targeting of these cells has been proven to be an effective method to enhance the phagocytosis of macrophages (Figure 4) (6). Knockdown of CD24 expression by CD24-shRNA can significantly inhibit cell viability and induce the apoptosis of SKOV3 cells (104). Administration with CD24-shRNA in vivo suppressed tumor volume increase by microvessel density (MVD) decrease, cell proliferation inhibition, and apoptosis induction, suggesting that knockdown of CD24 may be a potential method for the treatment of human ovarian cancer (104). When CD24 targeted siRNA molecules are added to the growth medium of several epithelial cancer cell lines, such as breast cancer and prostate cancer, the transient low expression of CD24 leads to the decrease of cell growth, and the changes of actin cytoskeleton can be observed, which results in exercise damage (18).

\section{The Binding of Targeting CD24-Siglec-10}

Siglec-10 is thought to have the ability to recognize sialic acid structures, and it binds firmly to CD24 in a sialic aciddependent manner $(43,44)$. The latest research shows that loss of tumor sialic acid can block the effect of immune modulatory Siglecs on immune cells (105). It has been reported that the antibody-sialidase conjugates are used to edit the glycocalyx accurately, and the antibody guides sialidase to selectively remove sialic acid from tumor cells, which enhances the sensitivity of tumor cells to antibody-dependent cellmediated cytotoxic (ADCC) and enables immune cells to kill desialylated cancer cells (106). For example, treating CD24 with sialidase abrogates the interaction with Siglec-10 and CD24 (Figure 4) (107). Barkal et al. also observed that the binding of Siglec-10 Fc (Fc, crystallizable fragments) to MCF-7 cells decreased significantly after desialylation on the cell surface (6). Blocking the interaction between CD24 and Siglec-10 with a monoclonal antibody can robustly augment the phagocytosis of human tumors expressing CD24 (6).

\section{Other}

CD24 on the surface of tumor cells can also be regulated by other factors to promote tumor immune escape. HIF-1a induces the expression of $\mathrm{CD} 24$ at the transcriptional level to promote tumor immune escape, and the non-coding RNA, Wnt/ $\beta$ catenin, promotes or inhibits the expression of CD24 to promote or inhibit tumor immune escape (61). Although the effects of hypoxia on tumor growth and metastasis have been known for a long time, recent studies show that hypoxia can also promote tumor immune escape. Current studies suggest that HIF-1 $\alpha$ can induce the expression of CD24 at the transcriptional level, 
which further points out the importance of hypoxia and the expression of CD24 for tumor immune escape (61). The decrease of HIF- $1 \alpha$ or CD2 4 expression mediated by shRNA reduces the survival rate of cancer cells in vivo and in vitro at the growth level of primary and metastatic tumors (Figure 4) (61). Downregulating HIF-1 can improve the sensitivity of chemotherapy and inhibit tumor formation. Therefore, inhibiting these CD24related upstream molecules of regulatory signaling pathways can effectively prevent tumor invasion and immune escape, improve the tumor microenvironment, and may have a positive effect on tumor treatment.

\section{REFERENCES}

1. Chen L, Flies DB. Molecular mechanisms of $\mathrm{T}$ cell co-stimulation and co-inhibition. Nat Rev Immunol. (2013) 13:227-42. doi: 10.1038/nri3405

2. Prendergast G. Immune escape as a fundamental trait of cancer: focus on IDO. Oncogene. (2008) 27:3889-900. doi: 10.1038/onc.2008.35

3. Topalian SL, Drake CG, Pardoll DM. Targeting the PD-1/B7-H1 (PD-L1) pathway to activate anti-tumor immunity. Curr Opin Immunol. (2012) 24:207-12. doi: 10.1016/j.coi.2011.12.009

4. Leach DR, Krummel MF, Allison JP. Enhancement of antitumor immunity by CTLA-4 blockade. Science. (1996) 271:17346. doi: $10.1126 /$ science.271.5256.1734

5. Willingham SB, Volkmer J-P, Gentles AJ, Sahoo D, Dalerba P, Mitra SS, et al. The CD47-signal regulatory protein alpha (SIRPa) interaction is a therapeutic target for human solid tumors. Proc Natl Acad Sci. (2012) 109:6662-7. doi: 10.1073/pnas.1121623109

6. Barkal AA, Brewer RE, Markovic M, Kowarsky M, Barkal SA, Zaro BW, et al. CD24 signalling through macrophage Siglec-10 is a target for cancer immunotherapy. Nature. (2019) 572:392-6. doi: 10.1038/s41586-019-1456-0

7. Springer T, Galfre G, Secher D, Milstein C. Monoclonal xenogeneic antibodies to murine cell surface antigens: identification of novel leukocyte differentiation antigens. Eur J Immunol. (1978) 8:539-51. doi: 10.1002/eji.1830080802

8. Crispe I, Bevan M. Expression and functional significance of the J11d marker on mouse thymocytes. J Immunol. (1987) 138:2013-8.

9. Hardy R, Hayakawa K, Parks D, Herzenberg L, Herzenberg L. Murine B cell differentiation lineages. J Exp Med. (1984) 159:1169-88. doi: 10.1084/jem.159.4.1169

10. Li O, Zheng P, Liu Y. CD24 expression on T cells is required for optimal $\mathrm{T}$ cell proliferation in lymphopenic host. J Exp Med. (2004) 200:10839. doi: 10.1084/jem.20040779

11. Eyvazi S, Kazemi B, Bandehpour M, Dastmalchi S. Identification of a novel single chain fragment variable antibody targeting CD24-expressing cancer cells. Immunol Lett. (2017) 190:240-6. doi: 10.1016/j.imlet.2017.08.028

12. Suzuki T, Kiyokawa N, Taguchi T, Sekino T, Katagiri YU, Fujimoto J. CD24 induces apoptosis in human B cells via the glycolipid-enriched membrane domains/rafts-mediated signaling system. J Immunol. (2001) 166:556777. doi: 10.4049/jimmunol.166.9.5567

13. Zarn JA, Zimmermann SM, Pass MK, Waibel R, Stahel RA. Association of CD24 with the kinase c-fgr in a small cell lung cancer cell line and with the kinase lyn in an erythroleukemia cell line. Biochem Biophys Res Commun. (1996) 225:384-91. doi: 10.1006/bbrc.1996.1184

14. Stefanova I, Horejsi V, Ansotegui I, Knapp W, Stockinger H. GPI-anchored cell-surface molecules complexed to protein tyrosine kinases. Science. (1991) 254:1016-9. doi: 10.1126/science.1719635

15. Baumann P, Cremers N, Kroese F, Orend G, Chiquet-Ehrismann R, Uede T, et al. CD24 expression causes the acquisition of multiple cellular properties associated with tumor growth and metastasis. Cancer Res. (2005) 65:1078393. doi: 10.1158/0008-5472.CAN-05-0619

16. Senner V, Sturm A, Baur I, Schrell UH, Distel L, Paulus W. CD24 promotes invasion of glioma cells in vivo. J Neuropathol Exp Neurol. (1999) 58:795802. doi: 10.1097/00005072-199908000-00002

\section{AUTHOR CONTRIBUTIONS}

F-HG handled the conceptualization and the writing and editing of the review. S-SY handled the writing of the original draft.

\section{FUNDING}

This work was supported in part by the National Natural Science Foundation of China (81172322, 81302006, and 81572796), Shanghai Municipal Education Committee (13ZZ089), and the Science and Technology Committee of Shanghai (14401901500).

17. Schabath H, Runz S, Joumaa S, Altevogt P. CD24 affects CXCR4 function in pre-B lymphocytes and breast carcinoma cells. J Cell Sci. (2006) 119:31425. doi: $10.1242 /$ jcs. 02741

18. Smith SC, Oxford G, Wu Z, Nitz MD, Conaway M, Frierson HF, et al. The metastasis-associated gene CD24 is regulated by Ral GTPase and is a mediator of cell proliferation and survival in human cancer. Cancer Res. (2006) 66:1917-22. doi: 10.1158/0008-5472.CAN-05-3855

19. Fukushima T, Tezuka T, Shimomura T, Nakano S, Kataoka H. Silencing of insulin-like growth factor-binding protein-2 in human glioblastoma cells reduces both invasiveness and expression of progression-associated gene CD24. J Biol Chem. (2007) 282:18634-44. doi: 10.1074/jbc.M609567200

20. Runz S, Mierke CT, Joumaa S, Behrens J, Fabry B, Altevogt P. CD24 induces localization of betal integrin to lipid raft domains. Biochem Biophys Res Commun. (2008) 365:35-41. doi: 10.1016/j.bbrc.2007.10.139

21. Sagiv E, Starr A, Rozovski U, Khosravi R, Altevogt P, Wang T, et al. Targeting CD24 for treatment of colorectal and pancreatic cancer by monoclonal antibodies or small interfering RNA. Cancer Res. (2008) 68:2803-12. doi: 10.1158/0008-5472.CAN-07-6463

22. Ahmed MA, Al-Attar A, Kim J, Watson NF, Scholefield JH, Durrant LG, et al. CD24 shows early upregulation and nuclear expression but is not a prognostic marker in colorectal cancer. J Clin Pathol. (2009) 62:111722. doi: 10.1136/jcp.2009.069310

23. Wang W, Wang X, Peng L, Deng Q, Liang Y, Qing H, et al. CD24-dependent MAPK pathway activation is required for colorectal cancer cell proliferation. Cancer Sci. (2010) 101:112-9. doi: 10.1111/j.1349-7006.2009.01370.x

24. Mierke CT, Bretz N, Altevogt P. Contractile forces contribute to increased glycosylphosphatidylinositol-anchored receptor CD24-facilitated cancer cell invasion. J Biol Chem. 286:34858-71. doi: 10.1074/jbc.M111. 245183

25. Bretz NP, Salnikov AV, Perne C, Keller S, Wang X, Mierke CT, et al. CD24 controls Src/STAT3 activity in human tumors. Cell Mol Life Sci. (2012) 69:3863-79. doi: 10.1007/s00018-012-1055-9

26. Kristiansen G, Denkert C, Schlüns K, Dahl E, Pilarsky C, Hauptmann $\mathrm{S}$. CD24 is expressed in ovarian cancer and is a new independent prognostic marker of patient survival. Am J Pathol. (2002) 161:121521. doi: 10.1016/S0002-9440(10)64398-2

27. Ikenaga N, Ohuchida K, Mizumoto K, Yu J, Kayashima T, Hayashi A, et al. Characterization of CD24 expression in intraductal papillary mucinous neoplasms and ductal carcinoma of the pancreas. Hum Pathol. (2010) 41:1466-74. doi: 10.1016/j.humpath.2010.04.004

28. Aigner S, Ramos CL, Hafezi-moghadam A, Lawrence MB, Friederichs J, Altevogt P, et al. CD24 mediates rolling of breast carcinoma cells on Pselectin. FASEB J. (1998) 12:1241-51. doi: 10.1096/fasebj.12.12.1241

29. Bretz N, Noske A, Keller S, Erbe-Hofmann N, Schlange T, Salnikov AV, et al. CD24 promotes tumor cell invasion by suppressing tissue factor pathway inhibitor-2 (TFPI-2) in a c-Src-dependent fashion. Clin Exp Metastasis. (2012) 29:27-38. doi: 10.1007/s10585-011-9426-4

30. Macauley MS, Crocker PR, Paulson JC. Siglec-mediated regulation of immune cell function in disease. Nat Rev Immunol. (2014) 14:65366. doi: 10.1038/nri3737

31. Crocker PR, Paulson JC, Varki A. Siglecs and their roles in the immune system. Nat Rev Immunol. (2007) 7:255-66. doi: 10.1038/nri2056 
32. Pillai S, Netravali IA, Cariappa A, Mattoo H. Siglecs and immune regulation. Ann Rev Immunol. (2012) 30:35792. doi: 10.1146/annurev-immunol-020711-075018

33. Doody GM, Justement LB, Delibrias CC, Matthews RJ, Lin J, Thomas $\mathrm{ML}$, et al. A role in B cell activation for CD22 and the protein tyrosine phosphatase SHP. Science. (1995) 269:242-4. doi: 10.1126/science.7618087

34. Taylor VC, Buckley CD, Douglas M, Cody AJ, Simmons DL, Freeman SD. The myeloid-specific sialic acid-binding receptor, CD33, associates with the protein-tyrosine phosphatases, SHP-1 and SHP-2. J Biol Chem. (1999) 274:11505-12. doi: 10.1074/jbc.274.17.11505

35. Neel BG, Gu H, Pao L. The 'Shp'ing news: SH2 domain-containing tyrosine phosphatases in cell signaling. Trends Biochem Sci. (2003) 28:28493. doi: 10.1016/S0968-0004(03)00091-4

36. Lorenz U. SHP-1 and SHP-2 in T cells: two phosphatases functioning at many levels. Immunol Rev. (2009) 228:34259. doi: 10.1111/j.1600-065X.2008.00760.x

37. Whitney G, Wang S, Chang H, Cheng KY, Lu P, Zhou XD, et al. A new siglec family member, siglec-10, is expressed in cells of the immune system and has signaling properties similar to CD33. Eur J Biochem. (2001) 268:608396. doi: 10.1046/j.0014-2956.2001.02543.x

38. Munday J, Kerr S, Ni J, Cornish AL, Zhang JQ, Nicoll G, et al. Identification, characterization and leucocyte expression of Siglec-10, a novel human sialic acid-binding receptor. Biochem J. (2001) 355:489-97. doi: 10.1042/bj3550489

39. Bandala-Sanchez E, Zhang Y, Reinwald S, Dromey JA, Lee BH, Qian J, et al. $T$ cell regulation mediated by interaction of soluble CD52 with the inhibitory receptor Siglec-10. Nat Immunol. (2013) 14:741-8. doi: 10.1038/ni.2610

40. Zhang P, Lu X, Tao K, Shi L, Li W, Wang G, et al. Siglec-10 is associated with survival and natural killer cell dysfunction in hepatocellular carcinoma. J Surgical Res. (2015) 194:107-13. doi: 10.1016/j.jss.2014.09.035

41. Sammar M, Siwetz M, Meiri H, Fleming V, Altevogt P, Huppertz B. Expression of CD24 and Siglec-10 in first trimester placenta: implications for immune tolerance at the fetal-maternal interface. Histochem Cell Biol. (2016) 147:565-74. doi: 10.1007/s00418-016-1531-7

42. Chen GY, Tang J, Zheng P, Liu Y. CD24 and Siglec-10 selectively repress tissue damage-induced immune responses. Science. (2009) 323:17225. doi: 10.1126/science.1168988

43. Abram CL, Lowell CA. Shp1 function in myeloid cells. J Leukoc Biol. (2017) 102:657-75. doi: 10.1189/jlb.2MR0317-105R

44. Dietrich J, Cella M, Colonna M. Ig-like transcript 2 (ILT2)/leukocyte Ig-like receptor 1 (LIR1) inhibits TCR signaling and actin cytoskeleton reorganization. J Immunol. (2001) 166:251421. doi: 10.4049/jimmunol.166.4.2514

45. Chen G-Y, Brown NK, Zheng P, Liu Y. Siglec-G/10 in self-nonself discrimination of innate and adaptive immunity. Glycobiology. (2014) 24:800-6. doi: 10.1093/glycob/cwu068

46. Chen X, Varki A. Advances in the biology and chemistry of sialic acids. Acs Chemical Biol. (2010) 5:163-76. doi: 10.1021/cb900266r

47. Sato C. Chain length diversity of sialic acids and its biological significance. Trends Glycosci Glycotechnol. (2004) 16:331-44. doi: 10.4052/tigg.16.331

48. Cohen M, Varki A. The sialome-far more than the sum of its parts. Omics A J Integrative Biol. (2010) 14:455-64. doi: 10.1089/omi.2009.0148

49. Corfield AP, Berry M. Glycan variation and evolution in the eukaryotes. Trends Biochem Sci. (2015) 40:351-9. doi: 10.1016/j.tibs.2015. 04.004

50. Sammar M, Aigner S, Altevogt P. Heat-stable antigen (mouse CD24) in the brain: dual but distinct interaction with P-selectin and L1. Biochim Biophys Acta. (1997) 1337:287-94. doi: 10.1016/S0167-4838(96)00177-X

51. Kleene $\mathrm{R}$, Yang $\mathrm{H}$, Kutsche $\mathrm{M}$, Schachner $\mathrm{M}$. The neural recognition molecule L1 is a sialic acid-binding lectin for CD24, which induces promotion and inhibition of neurite outgrowth. J Biol Chem. (2001) 276:21656-63. doi: 10.1074/jbc.M101790200

52. Bleckmann C, Geyer H, Lieberoth A, Splittstoesser F, Liu Y, Feizi T, et al. O-glycosylation pattern of CD24 from mouse brain. Biol Chem. (2009) 390:627-45. doi: 10.1515/BC.2009.044

53. Bleckmann C, Geyer H, Reinhold V, Lieberoth A, Schachner M, Kleene R, et al. Glycomic analysis of N-linked carbohydrate epitopes from CD24 of mouse brain. J Proteome Res. (2009) 8:567-82. doi: 10.1021/pr800729r
54. Büll C, Heise T, Adema GJ, Boltje TJ. Sialic acid mimetics to target the sialic acid-Siglec axis. Trends Biochem Sci. (2016) 41:519-31. doi: 10.1016/j.tibs.2016.03.007

55. Kelm S, Schauer R, Manuguerra J-C, Gross H-J, Crocker PR. Modifications of cell surface sialic acids modulate cell adhesion mediated by sialoadhesin and CD22. Glycoconjugate J. (1994) 11:576-85. doi: 10.1007/BF00731309

56. Sjoberg ER, Powell LD, Klein A, Varki A. Natural ligands of the B cell adhesion molecule CD22 beta can be masked by 9-O-acetylation of sialic acids. J Cell Biol. (1994) 126:549-62. doi: 10.1083/jcb.126.2.549

57. Bardor M, Nguyen DH, Diaz S, Varki A. Mechanism of uptake and incorporation of the non-human sialic acid N-glycolylneuraminic acid into human cells. J Biol Chem. (2005) 280:4228-37. doi: 10.1074/jbc.M412040200

58. Jiang X, Wang J, Deng X, Xiong F, Ge J, Xiang B, et al. Role of the tumor microenvironment in $\mathrm{PD}-\mathrm{L} 1 / \mathrm{PD}-1$-mediated tumor immune escape. $\mathrm{Mol}$ Cancer. (2019) 18:10. doi: 10.1186/s12943-018-0928-4

59. Wong W, Goehring AS, Kapiloff MS, Langeberg LK, Scott JD. mAKAP compartmentalizes oxygen-dependent control of HIF-1? Sci Signaling. (2008) 1:ra18. doi: 10.1126/scisignal.2000026

60. Maxwell PH, Wiesener MS, Chang G-W, Clifford SC, Vaux EC, Cockman $\mathrm{ME}$, et al. The tumour suppressor protein VHL targets hypoxiainducible factors for oxygen-dependent proteolysis. Nature. (1999) 399:2715. doi: 10.1038/20459

61. Thomas S, Harding MA, Smith SC, Overdevest JB, Nitz MD, Frierson HF, et al. CD24 is an effector of HIF-1-driven primary tumor growth and metastasis. Cancer Res. (2012) 72:5600-12. doi: 10.1158/0008-5472.CAN-11-3666

62. Muppala S, Mudduluru G, Leupold JH, Buergy D, Sleeman JP, Allgayer H. CD24 induces expression of the oncomir miR-21 via Src, and CD24 and Src are both post-transcriptionally downregulated by the tumor suppressor miR-34a. PLoS ONE. (2013) 8:e59563. doi: 10.1371/journal.pone.0059563

63. Li X, Xin S, He Z, Che X, Wang J, Xiao X, et al. MicroRNA-21 (miR-21) posttranscriptionally downregulates tumor suppressor PDCD4 and promotes cell transformation, proliferation, and metastasis in renal cell carcinoma. Cell Physiol Biochem. (2014) 33:1631-42. doi: 10.1159/000362946

64. Meng F, Henson R, Wehbe-Janek H, Ghoshal K, Jacob ST, Patel T. MicroRNA-21 regulates expression of the PTEN tumor suppressor gene in human hepatocellular cancer. Gastroenterology. (2007) 133:64758. doi: 10.1053/j.gastro.2007.05.022

65. Scheurer SB, Raybak JN, Rösli C, Neri D, Elia G. Modulation of gene expression by hypoxia in human umbilical cord vein endothelial cells: a transcriptomic and proteomic study. Proteomics. (2004) 4:173760. doi: 10.1002/pmic.200300689

66. Fujikuni N, Yamamoto H, Tanabe K, Naito Y, Sakamoto N, Tanaka Y, et al. Hypoxia-mediated CD 24 expression is correlated with gastric cancer aggressiveness by promoting cell migration and invasion. Cancer Sci. (2014) 105:1411-20. doi: 10.1111/cas.12522

67. Patel JS, Hu M, Sinha G, Walker ND, Sherman LS, Gallagher A, et al. Non-coding RNA as mediators in microenvironmentbreast cancer cell communication. Cancer Lett. (2016) 380:28995. doi: 10.1016/j.canlet.2015.11.016

68. D'Asti E, Chennakrishnaiah S, Lee TH, Rak J. Extracellular vesicles in brain tumor progression. Cell Mol Neurobiol. (2016) 36:383-407. doi: 10.1007/s10571-015-0296-1

69. Sasaki N, Toyoda M, Yoshimura H, Matsuda Y, Arai T, Takubo K, et al. H19 long non-coding RNA contributes to sphere formation and invasion through regulation of CD24 and integrin expression in pancreatic cancer cells. Oncotarget. (2018) 9:34719. doi: 10.18632/oncotarget.26176

70. Fabian MR, Sonenberg N. The mechanics of miRNA-mediated gene silencing: a look under the hood of miRISC. Nat Struct Mol Biol. (2012) 19:586. doi: 10.1038/nsmb.2296

71. Ghuwalewala S, Ghatak D, Das S, Das P, Butti R, Gorain M, et al. MiR-146a-dependent regulation of CD24/AKT/ $\beta$-catenin axis drives cancer stem cell phenotype in oral squamous cell carcinoma. bioRxiv. (2019) 429068. doi: 10.1101/429068

72. Ahmad F, Dina K, Faina B, Eli B, Chen V. CD24 induces the activation of $\beta$-catenin in intestinal tumorigenesis. J Cancer Sci Ther. (2016) 8:13542. doi: 10.4172/1948-5956.1000405 
73. Shulewitz M, Soloviev I, Wu T, Koeppen H, Polakis P, Sakanaka C. Repressor roles for TCF-4 and Sfrp1 in Wnt signaling in breast cancer. Oncogene. (2006) 25:4361-9. doi: 10.1038/sj.onc. 1209470

74. Li Y, Zhou J, Zhuo Q, Zhang J, Xie J, Han S, et al. Malignant ascite-derived extracellular vesicles inhibit $\mathrm{T}$ cell activity by upregulating Siglec-10 expression. Cancer Manag Res. (2019) 11:7123-34. doi: 10.2147/CMAR.S210568

75. Zhao Y, Su H, Shen X, Du J, Zhang X, Zhao Y. The immunological function of CD52 and its targeting in organ transplantation. Inflamm Res. (2017) 66:571-8. doi: 10.1007/s00011-017-1032-8

76. Liu YC, Yu MM, Chai YF, Shou ST. Sialic acids in the immune response during sepsis. Front Immunol. (2017) 8:1601. doi: 10.3389/fimmu.2017.01601

77. Bärenwaldt A, Läubli H. The sialoglycan-Siglec glyco-immune checkpoint-a target for improving innate and adaptive anti-cancer immunity. Expert Opin Therapeutic Targets. (2019) 23:839-53. doi: 10.1080/14728222.2019.1667977

78. Meyer SJ, Linder AT, Brandl C, Nitschke L. B cell siglecs-news on signaling and its interplay with ligand binding. Front Immunol. (2018) 9:2820. doi: 10.3389/fimmu.2018.02820

79. Angata T, Hingorani R, Varki NM, Varki A. Cloning and characterization of a novel mouse siglec. mSiglec-F. (2001) 276:45128-36. doi: 10.1074/jbc.M108573200

80. McGary KL, Park TJ, Woods JO, Cha HJ, Wallingford JB, Marcotte EM. Systematic discovery of non-obvious human disease models through orthologous phenotypes. Proc Natl Acad Sci USA. (2010) 107:65449. doi: 10.1073/pnas.0910200107

81. Muller J, Szklarczyk D, Julien P, Letunic I, Roth A, Kuhn M, et al. eggNOG v2. 0: extending the evolutionary genealogy of genes with enhanced nonsupervised orthologous groups, species and functional annotations. Nucl Acids Res. (2010) 38:D190-5. doi: 10.1093/nar/gkp951

82. Gogarten JP, Olendzenski L. Orthologs, paralogs and genome comparisons. Curr Opin Genet Dev. (1999) 9:630-6. doi: 10.1016/S0959-437X(99)00029-5

83. Hoffmann A, Kerr S, Jellusova J, Zhang J, Weisel F, Wellmann U, et al. Siglec-G is a B1 cell-inhibitory receptor that controls expansion and calcium signaling of the B1 cell population. Nat Immunol. (2007) 8:695704. doi: $10.1038 /$ ni1480

84. Rodgers W, Crise B, Rose JK. Signals determining protein tyrosine kinase and glycosyl-phosphatidylinositol-anchored protein targeting to a glycolipid-enriched membrane fraction. Mol Cell Biol. (1994) 14:538491. doi: 10.1128/MCB.14.8.5384

85. Janes PW, Ley SC, Magee AI. Aggregation of lipid rafts accompanies signaling via the $\mathrm{T}$ cell antigen receptor. J Cell Biol. (1999) 147:44761. doi: $10.1083 /$ jcb.147.2.447

86. Nagasawa T, Nakajima T, Tachibana K, Iizasa H, Bleul CC, Yoshie O, et al. Molecular cloning and characterization of a murine pre-B-cell growthstimulating factor/stromal cell-derived factor 1 receptor, a murine homolog of the human immunodeficiency virus 1 entry coreceptor fusin. Proc Natl Acad Sci. (1996) 93:14726-9. doi: 10.1073/pnas.93.25.14726

87. Taguchi T, Kiyokawa N, Mimori K, Suzuki T, Sekino T, Nakajima $\mathrm{H}$, et al. Pre-B cell antigen receptor-mediated signal inhibits CD24induced apoptosis in human pre-B cells. J Immunol. (2003) 170:25260. doi: 10.4049/jimmunol.170.1.252

88. Jutras I, Desjardins M. Phagocytosis: at the crossroads of innate and adaptive immunity. Annu Rev Cell Dev Biol. (2005) 21:51127. doi: 10.1146/annurev.cellbio.20.010403.102755

89. Chao MP, Jaiswal S, Weissman-Tsukamoto R, Alizadeh AA, Gentles AJ, Volkmer J, et al. Calreticulin is the dominant pro-phagocytic signal on multiple human cancers and is counterbalanced by CD47. Sci Transl Med. (2010) 2:63. doi: 10.1126/scitranslmed.3001375

90. Chen J, Zhong M-C, Guo H, Davidson D, Mishel S, Lu Y, et al. SLAMF7 is critical for phagocytosis of haematopoietic tumour cells via Mac-1 integrin. Nature. (2017) 544:493-7. doi: 10.1038/nature22076

91. Veillette A, Chen J. SIRP $\alpha$-CD47 immune checkpoint blockade in anticancer therapy. Trends Immunol. (2018) 39:173-84. doi: 10.1016/j.it.2017.12.005
92. Gordon SR, Maute RL, Dulken BW, Hutter G, George BM, McCracken $\mathrm{MN}$, et al. PD-1 expression by tumour-associated macrophages inhibits phagocytosis and tumour immunity. Nature. (2017) 545:495-9. doi: 10.1038/nature22396

93. Barkal AA, Weiskopf K, Kao KS, Gordon SR, Rosental B, Yiu YY, et al. Engagement of MHC class I by the inhibitory receptor LILRB1 suppresses macrophages and is a target of cancer immunotherapy. Nat Immunol. (2018) 19:76-84. doi: 10.1038/s41590-017-0004-Z

94. Chen H-M, van der Touw W, Wang YS, Kang K, Mai S, Zhang J, et al. Blocking immunoinhibitory receptor LILRB2 reprograms tumor-associated myeloid cells and promotes antitumor immunity. J Clin Investig. (2018) 128:5647-62. doi: 10.1172/JCI97570

95. Jaiswal S, Jamieson CH, Pang WW, Park CY, Chao MP, Majeti R, et al. CD47 is upregulated on circulating hematopoietic stem cells and leukemia cells to avoid phagocytosis. Cell. (2009) 138:271-85. doi: 10.1016/j.cell.2009.05.046

96. Zhang J, Somani A-K, Siminovitch KA. Roles of the SHP-1 tyrosine phosphatase in the negative regulation of cell signalling. Semin Immunol. (2000) 12:361-78. doi: 10.1006/smim.2000.0223

97. Oldenborg P-A, Zheleznyak A, Fang Y-F, Lagenaur CF, Gresham HD, Lindberg FP. Role of CD47 as a marker of self on red blood cells. Science. (2000) 288:2051-4. doi: 10.1126/science.288.5473.2051

98. Barclay AN, Van den Berg TK. The interaction between signal regulatory protein alpha (SIRPr $\alpha \mathrm{r}$ ) and CD47: structure, function, and therapeutic target. Ann Rev Immunol. (2014) 32:25-50. doi: 10.1146/annurev-immunol-032713-120142

99. Medzhitov R, Janeway CA. Decoding the patterns of self and non-self by the innate immune system. Science. (2002) 296:298-300. doi: 10.1126/science.1068883

100. Chao MP, Weissman IL, Majeti R. The CD47-SIRP $\alpha$ pathway in cancer immune evasion and potential therapeutic implications. Curr Opin Immunol. (2012) 24:225-32. doi: 10.1016/j.coi.2012.01.010

101. Feng M, Jiang W, Kim BY, Zhang CC, Fu Y-X, Weissman IL. Phagocytosis checkpoints as new targets for cancer immunotherapy. Nat Rev Cancer. (2019) 19:568-86. doi: 10.1038/s41568-019-0183-z

102. Jellusova J, Duber S, Guckel E, Binder CJ, Weiss S, Voll R, et al. Siglec-G regulates B1 cell survival and selection. J Immunol. 185:327784. doi: 10.4049/jimmunol.1001792

103. Salnikov AV, Bretz NP, Perne C, Hazin J, Keller S, Fogel M, et al. Antibody targeting of CD24 efficiently retards growth and influences cytokine milieu in experimental carcinomas. Br J Cancer. (2013) 108:144959. doi: 10.1038/bjc.2013.102

104. Su D, Deng H, Zhao X, Zhang X, Chen L, Chen X, et al. Targeting CD24 for treatment of ovarian cancer by short hairpin RNA. Cytotherapy. (2009) 11:642-52. doi: 10.1080/14653240902878308

105. Munkley J, Scott E. Targeting aberrant sialylation to treat cancer. Medicines. (2019) 6:102. doi: 10.3390/medicines6040102

106. Xiao H, Woods EC, Vukojicic P, Bertozzi CR. Precision glycocalyx editing as a strategy for cancer immunotherapy. Proc Natl Acad Sci USA. (2016) 113:10304-9. doi: 10.1073/pnas.1608069113

107. Chen GY, Chen X, King S, Cavassani KA, Cheng J, Zheng X, et al. Amelioration of sepsis by inhibiting sialidase-mediated disruption of the CD24-SiglecG interaction. Nat Biotechnol. (2011) 29:428-35. doi: 10.1038/nbt.1846

Conflict of Interest: The authors declare that the research was conducted in the absence of any commercial or financial relationships that could be construed as a potential conflict of interest.

Copyright (C) 2020 Yin and Gao. This is an open-access article distributed under the terms of the Creative Commons Attribution License (CC BY). The use, distribution or reproduction in other forums is permitted, provided the original author(s) and the copyright owner(s) are credited and that the original publication in this journal is cited, in accordance with accepted academic practice. No use, distribution or reproduction is permitted which does not comply with these terms. 